\title{
Survival Analysis of CABG Patients by Parametric Estimations In Modifiable Risk Factors - Hypertension and Diabetes
}

\author{
M. Saleem ${ }^{1, *}$, Zafar Mahmud ${ }^{2}$, K. H. Khan ${ }^{3}$ \\ ${ }^{1}$ Centre for Advanced Studies in Pure and Applied Mathematics Bahauddin Zakariya University Multan, Pakistan \\ ${ }^{2}$ Department of Mathematics: COMSATS Attock, Pakistan \\ ${ }^{3}$ Department of Mathematics, College of Science and Humanities, Salman Bin AbdulAziz University, Al Kharj, Saudi Arabia
}

\begin{abstract}
In this paper, modifiable risk factors (hypertension and diabetes) of Coronary Artery Bypass Graft Surgery (CABG) patients are considered. The objective is to analyse survivor's proportions of CABG patients in the considered risk factors, in complete and incomplete Populations, using suitable survival models. A new approach of complete population from its incomplete population of CABG patients of 12 years observations is used for the survival analysis. In the complete population, censored patients are proportionally included into the known survived and died patients respectively. The availability of a complete population may represent better behaviour of lifetimes / survival proportions for medical research. Survival proportions of the CABG patients of complete and incomplete populations, with respect to the risk factors (hypertension and diabetes) are obtained from suitable, lifetime representing models (Weibull and Exponential). Maximum likelihood method, in-conjunction with Davidon-Fletcher-Powell (DFP) optimization method and Cubic Interpolation method is used in estimation of survivor's proportions from the parametric models.
\end{abstract}

Keywords CABG Patients, Complete \& Incomplete Populations, Modifiable Risk Factors, Parametric survival models (Weibull and Exponential), Maximum likelihood method, Davidon-Fletcher-Powell optimization method and Survivor's Proportions

\section{Introduction}

The Coronary Artery Disease (CAD) is due to accumulation of cholesterol and other material, called plaque, within inner walls of the coronary arteries (the arteries that supply blood with oxygen and nutrients to heart muscles). As this build-up grows (Arteriosclerosis), comparatively less blood can flow through the arteries. Over the time (which differs from individual to individual) heart weakens. This leads to chest pain (Angina) which is a symptom of Myocardial Infarction (MI). When the clot (thrombus) completely cuts off the hearts blood supply, this may leads to permanent heart damage, called as heart attack (MI). Heart failure means the heart cannot pump required blood to the rest of the body[10]. CAD is the leading cause of death worldwide (see William, Stephen, Van -Thomas and Robert)[36], John[18], Hansson[13], A xel, Yiwen, Dalit, Veena, Elaine, Catia, Matthew, Jonathan, Edward \& Len[3] and Sun \& Hoong[33] ). The symptoms and signs of

* Corresponding author:

colsaleem_2009@y ahoo.com (M. Saleem)

Published online at http://journal.sapub.org/ajms

Copyright @ 2012 Scientific \& Academic Publishing. All Rights Reserved coronary artery disease are noted in the advanced stage of the disease. Initially, most of the individuals with CAD show no evidence of disease, whereas the disease progresses before the first onset of symptoms and often there is a sudden heart attack. The CAD is the most common cause of sudden death of men and wo men over 20 years of age[30]. The medical scientists; William, Ellis, Josef, Ralph and Robert [36], Heymann[15], Goldstein, Adams, Alberts, Appel, Brass, Bushnell, Culebras, DeGraba, Gorelick \& Guyton[11] and Jennifer[17] are of the opinion that CABG is an effective treatment option for CAD patients. The medical research organizations like Heart and Stroke Foundation Canada[14] and American Heart Association[2] have classified risk factors of CABG patients as modifiable and non-modifiable. Modifiable risk factors are those factors, which may be controlled by changing life style or taking medications to reduce cardiovascular risk. These risk factors include high blood pressure (hypertension), diabetes, smoking, high cholesterol, sedentary lifestyle and obesity. Non-modifiable risk factors are those factors, which may not be changed. These factors include age, gender and family history (Genetic predisposition). William, Ellis, Josef, Ralph and Robert[35] in 1995 carried out the survival study on incomp lete population (progressive censoring of type 1) of 
CABG patients comprising 2011 patients using Kaplan Meier method[19]. The patients were grouped with respect to Male, Female, Age, Hypertension, Diabetes, and Ejection Fraction, Vessels, Congestive Heart Failure, Elective and Emergency Surgery. The patients were undergone through a first re-operation at Emory University hospitals from 1975 to 1993. This study also comprises the same data set of 2011 patients. The details of patients are given in the article[35].

In this paper we present survival analysis of the CABG patients with respect to some modifiable risk factors, Hypertension \& Diabetes, in complete and incomplete Populations. Khan, Saleem and Mahmud in the article[21] proposed a procedure, to make an incomplete population (IP) a complete population $(C P)$. The differences between the means of survival proportions of the CABG patients, obtained by using survival models (Weibull, Exponential etc) are statistically insignificant at $5 \%$ level of significance for details see the article[21].

The importance of parametric models for analysis of lifetime date has been indicated by Mann, Schefer and Singpurwala[28], Nelson[29], Cyrus[8], Lawless[25], Klein \& Moeschberger[22] and Sridhar and Mun Choon Chan[32]. The Exponential distribution model has been used by Lee, Kim and Jung[27] in medical research for survival data of patients. The Weibull distribution model has been used for survival analysis by Cohen[6], Gross and Clark[12], Bunday[5], Crow[7], Klein \& Moeschberger[22], Lawrencce[26], Abrenthy[1], Hisada \& Arizino[16], Lawless[25], David \& Mitchel[9] and Lang[23]. In particular, the survival study of chronic diseases, such as AIDS and Cancer, has been carried out by Bain and Englehardt[4], Khan \& Mahmud[20], Klein \& Moeschberger[22], Lawless[25] and Swaminathan and Brenner[34] using Exponential and Weibull distributions. Lanju \& William[24] used Weibull distribution to human survival data of patients with plas ma cell and in response-adaptive randomization for survival trials respectively. Lee, Kim and Jung[27] used the exponential in medical research for survival data of the patients. Khan, Saleem and Mohmud[21] concluded that the survival data of the CABG patients has been best modeled by the Weibull and Exponential distributions. In this paper, the survivor proportions of the CABG patients are obtained for incomplete and complete populations of the CABG patients by parametric models (Weibull and Exponential), using data of CABG patients Hypertension and Diabetes. Maximum likelihood method, in-conjunction with DFP optimization method and Cubic Interpolation method is used. A subroutine for maximizing log-likelihood function of each model is developed in FORTRAN program to obtain the estimates of the parameters of the model. The survival proportions of IP and $C P$ of the CABG patients with respect to modifiable risk factors are presented in term of statistics and graphs (survival curves), discussed and concluded.

\section{Methodology}

Khan, Saleem and Mahmud mentioned that the method proposed by Kaplan Meier[19] and latter discussed by William[35] in 1995 and Lawless[25] is: $S(t)=\prod_{j: t_{j}<t}\left(1-\frac{d_{j}}{n_{j}}\right)$, where $d_{j}$ and $n_{j}$ are the number of items (individuals / patients) failed (died individuals) and number of individuals at risk at time $t_{j}$, that is, the number of individuals survived and uncensored at time $t_{j-1}$. This method does not take into account the censored individuals $c_{j}$ completely and thus the analysis is performed on incomplete population (IP) . Further, Khan, Saleem and Mahmud[21] proposed that the censored individuals $C_{j}$ could be taken into account. The inclusion of splitted-censored individuals, $c_{j}$ proportionally $\left[\left(1-\frac{d_{j}}{n_{j-1}-c_{j}}\right) \times c_{j}\right.$ and $\left.\left(\frac{d_{j}}{n_{j-1}-c_{j}}\right) \times c_{j}\right]$ into known survived, $n_{j}$ and died individual's $d_{j}$ respectively makes the population complete. Thus the survival analys is may be performed on the complete population $(C P)$ also. Saleem, Mahmud and Khan[31] mentioned the form of likelihood function proposed by Klein \& Moeschberger[22] and Lawless[25], for a survival model, in the presence of censored data. The maximum likelihood method works by developing a likelihood function based on the available data and finding the estimates of parameters of a probability distribution that maximizes the likelihood function. This may be achieved by using iterative method: see Bunday \& Al-Mutwali[5] and Khan \& Mahmud[20] The likelihood function for all observed died and censored individuals is of the form: $L(t ; \underline{\theta})=\prod_{i=1}^{n}\left[f\left(t_{i} ; \underline{\theta}\right)\right]^{f_{t_{i}}} \prod_{i=1}^{n}\left[S\left(t_{i} ; \underline{\theta}\right)\right]^{{ }^{c_{t_{i}}}}$, where $f_{t_{i}} \& C_{t_{i}}$ are the number of died \& censored individuals in interval $i$ each of length $t, f(t ; \underline{\theta})$ is $p d f$ in a parametric model with survivor function, $S(t ; \underline{\theta})$ \& hazard function, $h(t ; \underline{\theta})$ and $\underline{\theta}$ is vector of parameters say $\underline{\theta}=(\alpha, \beta)$ of the model . To obtain maximum likelihood estimates of parameters of a parametric model using DFP optimization method, we take negative log on both the sides of above equation and therefore by setting $l=-\ln (L(t ; \underline{\theta}))$, we get:

$$
\begin{aligned}
& l=-\sum_{i=1}^{n} f_{t_{i}} \ln f\left(t_{i} ; \underline{\theta}\right)-\sum_{i=1}^{n} c_{t_{i}} \ln \left(S\left(t_{i} ; \underline{\theta}\right)\right) \text { or } \\
& =-\sum_{i=1}^{n} f_{t_{i}} \ln h\left(t_{i} ; \underline{\theta}\right)-\sum_{i=1}^{n}\left(f_{t_{i}}+c_{t_{i}}\right) \ln \left(S\left(t_{i} ; \underline{\theta}\right)\right)
\end{aligned}
$$




$$
\text { (As } \left.f\left(t_{i} ; \underline{\theta}\right)=h\left(t_{i} ; \underline{\theta}\right) S\left(t_{i} ; \underline{\theta}\right)\right)
$$

Where, the first sum is for failure and the second sum is for all censored individuals.

Setting $N_{t_{i}}=\left(f_{t_{i}}+c_{t_{i}}\right)$, where $N_{t_{i}}$ represents total no of individuals at time $t_{i}$ we get:

$$
l=-\sum_{i=1}^{n} f_{t_{i}} \ln h\left(t_{i} ; \underline{\theta}\right)-\sum_{i=1}^{n}\left(N_{t_{i}}\right) \ln \left(S\left(t_{i} ; \underline{\theta}\right)\right)
$$

In this study time is partitioned into intervals, which are of unit length $t$ starting from zero. Moreover, failures and censoring of the patients occur in each interval $i$ of equal length of time $t, \quad i=1,2, \ldots, 12$.

For complete population the term for censored observations is dropped from the likelihood function.

\section{Application}

Khan, Saleem and Mahmud[21] presented detail application of above methodology for parametric model (Weibull distribution). Same procedure is followed for second parametric model (Exponential distribution with $\beta$ $=1$ ) considered in this article. The methodology is reproduced here. The probability density function ( $p d f)$ of Weibull distribution is:

$$
f(t ; \underline{\theta})=\left(\frac{\beta}{\alpha}\right)\left(\frac{t}{\alpha}\right)^{\beta-1} e^{-\left(\frac{t}{\alpha}\right)^{\beta}},
$$

where $\underline{\theta}$ is vector of parameters $\alpha$ and $\beta$; $\alpha$ is a scale parameter and $\beta$ is a shape parameter; $\alpha, \beta$ and $t>0$.

The survival and hazard functions of Weibull distribution

are: $S(t ; \underline{\theta})=e^{-\left(\frac{t}{\alpha}\right)^{\beta}}$

and $h(t ; \underline{\theta})=\left(\frac{\beta}{\alpha}\right)\left(\frac{t}{\alpha}\right)^{\beta-1}$

For inco mplete population replacing values of the survival and hazard functions of Weibull distribution in equation (1), we get

$$
\begin{aligned}
& l=-\sum_{i=1}^{n} f_{t_{i}} \ln \left[\left(\frac{\beta}{\alpha}\right)\left(\frac{t_{i}}{\alpha}\right)^{\beta-1}\right] \\
& -\sum_{i=1}^{n} N_{t_{i}} \ln \left(e^{-\left(\frac{t_{i}}{\alpha}\right)^{\beta}}\right)
\end{aligned}
$$

or

$$
\begin{aligned}
& =-(F) \ln \left(\frac{\beta}{\alpha}\right) \\
& -(\beta-1) \sum_{i=1}^{n} f_{t_{i}} \ln \left(\frac{t_{i}}{\alpha}\right)+\sum_{i=1}^{n} N_{t_{i}}\left(\frac{t_{i}}{\alpha}\right)^{\beta},
\end{aligned}
$$

where, $F=\sum_{i=1}^{n} f_{t_{i}}$ is the total number of failures in a given time.

Differentiating (2) with respect to $\alpha$ and $\beta$ and simp lifying we get

$$
\begin{gathered}
\frac{\partial l}{\partial \alpha}=F\left(\frac{\beta}{\alpha}\right)-\left(\frac{\beta}{\alpha}\right) \sum_{i=1}^{n} N_{t_{i}}\left(\frac{t_{i}}{\alpha}\right)^{\beta} \\
\frac{\partial l}{\partial \beta}=-\frac{F}{\beta}-\sum_{i=1}^{n} f_{t_{i}} \ln \left(\frac{t_{i}}{\alpha}\right) \\
+\sum_{i=1}^{n} N_{t_{i}}\left(\frac{t_{i}}{\alpha}\right)^{\beta} \ln \left(\frac{t_{i}}{\alpha}\right)
\end{gathered}
$$

By using (2), (3) and (4) in the DFP optimization method, we find the parameters estimates for which value of the likelihood function is maximum. For complete population we drop the term for censored observations from likelihood function. Same procedure is followed for Exponential model. FORTRAN program for the parameters estimation of both the models is developed. The optimal estimates of the scale and shape / location parameters ( $\alpha$ and $\beta$ respectively) of Weibull and Exponential distributions distribution using $C P$ of groups: Hypertension (Absent \& Present), Diabetes (Absent \& Present) are obtained by maximizing the log-likelihood function. The $t$-ratios of the parameters are given in parenthesis. The values of parameters estimates, $t$-ratios, log-likelihood function and variance-covariance matrix are given below: -

\section{Weibull and Exponential Distributions using Data of Modifiable Risk Factors}

\subsection{Hypertension Absent (HYa) and Present (HYp) Groups (Male and Female CABG Patients)}

The survival proportions $\hat{y}_{t}$ and $y / c$ of $C P$ and $I P$ respectively of $H Y_{a}$ and $H Y_{p}$ groups of CABG patients are obtained using Weibull and exponential distributions as explained earlier. The optimal estimates of parameters obtained by maximizing the log-likelihood function are given below in tables 1 and 2 .

The estimated values of scale parameter $\alpha>0$ and shape parameter $\beta>0$ for $C P$ and $I P$ of $H Y_{a}$ and $H Y_{p}$ groups of CABG patients are given in the tables 1 and 2 along with $t$-ratios in the parenthesis, indicating that the estimates of scale and shape parameters are significant at $5 \%$ level of significance. In case of weibull distribution the estimated value of $\beta$ is greater than 1 (for $C P$ and $I P$ of the $H Y_{a}$ and $H Y_{p}$ groups) which indicates increasing failure rate 
with time. The negative values of co-variances (for $C P$ and IP of $H Y_{a}$ and $H Y_{p}$ groups of CABG patients) indicates that the movements of $\hat{\alpha}$ and $\hat{\beta}$ are in the opposite directions.

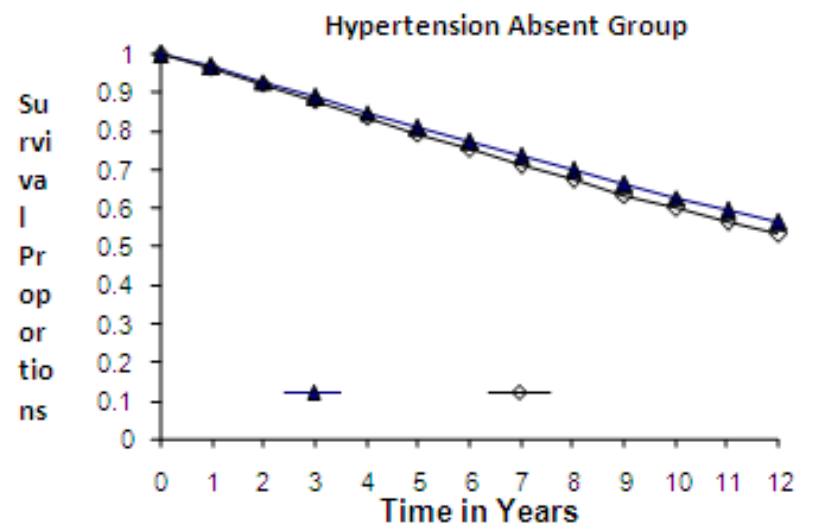

Figure 1. Weibull Distribution

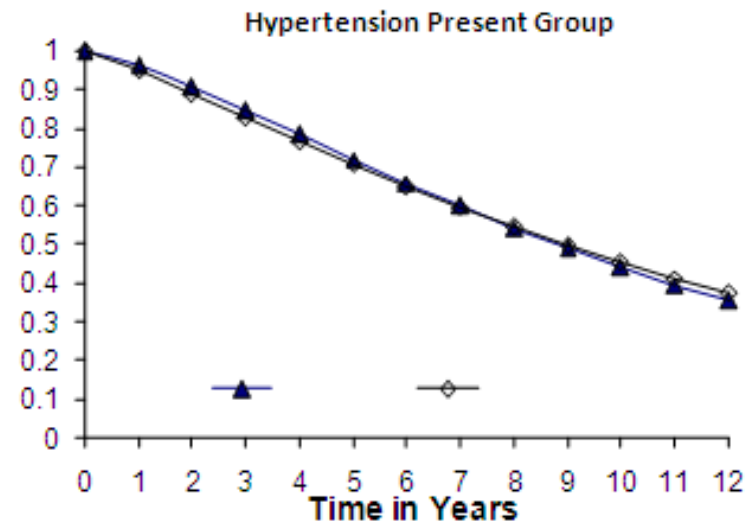

Figure 2. Weibull Distribution

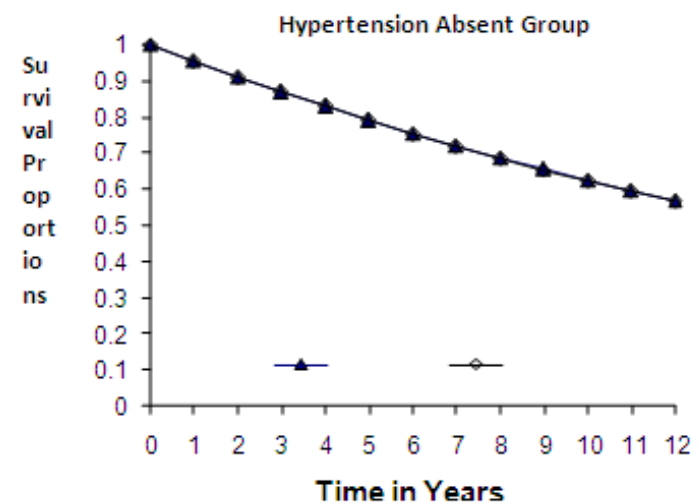

Figure 3. Exponential Distribution

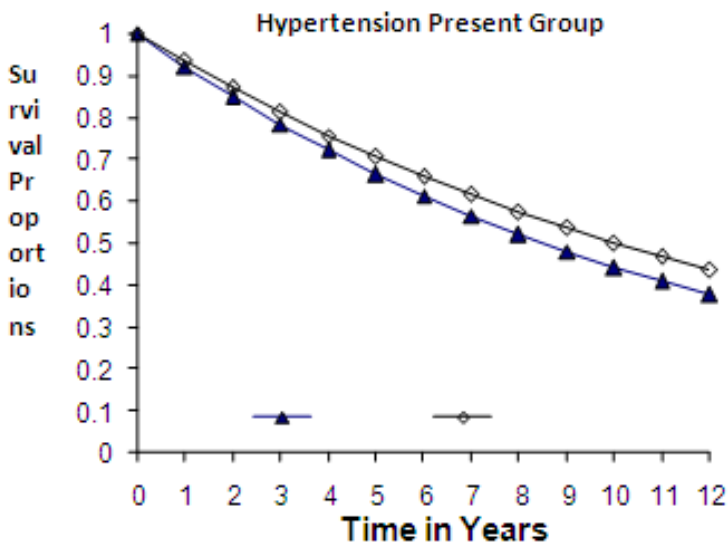

Figure 4. Exponential Distribution

The values of estimated survival proportions of $C P$ and $I P$ of $H Y_{a}$ and $H Y_{p}$ groups of CABG patients are given in table 3 - and corresponding graphs (survival curves) in fig 1 to 4 .

Table 1. Estimates of Paramet ers of Weibull Distribution Using Data of $C P$ and $I P$ of $H Y_{a}$ and $H Y_{p}$ Groups of CABG patients

\begin{tabular}{|c|c|c|c|c|c|c|c|c|}
\hline \multirow{3}{*}{ Parameters } & \multicolumn{4}{|c|}{$H Y_{a}$ Group } & \multicolumn{4}{|c|}{$H Y_{P}$ Group } \\
\hline & \multicolumn{2}{|c|}{$C P$} & \multicolumn{2}{|c|}{$I P$} & \multicolumn{2}{|c|}{$C P$} & \multicolumn{2}{|c|}{$I P$} \\
\hline & $\begin{array}{l}\text { Estimates/ } \\
\text { (t-ratio) }\end{array}$ & Gradients & $\begin{array}{l}\text { Estimates/ } \\
\text { (t-ratio) }\end{array}$ & Gradients & $\begin{array}{l}\text { Estimates/ } \\
\text { (t-ratio) }\end{array}$ & Gradients & $\begin{array}{c}\begin{array}{c}\text { Estimates/ } \\
\text { (t-ratio) }\end{array} \\
\end{array}$ & Gradients \\
\hline$\alpha$ & $\begin{array}{l}19.476 \\
(19.92) \\
\end{array}$ & $2.48 \times 10^{-10}$ & $\begin{array}{l}17.766 \\
(11.13) \\
\end{array}$ & $1.39 \times 10^{-9}$ & $\begin{array}{l}11.659 \\
(31.73) \\
\end{array}$ & $-1.21 \times 10^{-9}$ & $\begin{array}{l}12.188 \\
(14.18) \\
\end{array}$ & $1.15 \times 10^{-8}$ \\
\hline$\beta$ & $\begin{array}{c}1.142 \\
(22.14)\end{array}$ & $-6.22 \times 10^{-10}$ & $\begin{array}{l}1.150 \\
(16.94)\end{array}$ & $2.98 \times 10^{-8}$ & $\begin{array}{c}1.316 \\
(26.81)\end{array}$ & $-7.1 \times 10^{-8}$ & $\begin{array}{c}1.191 \\
(18.78)\end{array}$ & $8.49 \times 10^{-8}$ \\
\hline Log-Likelihood & \multicolumn{2}{|c|}{1743.991} & \multicolumn{2}{|c|}{819.2172} & \multicolumn{2}{|c|}{2054.818} & \multicolumn{2}{|c|}{871.8191} \\
\hline $\begin{array}{c}\text { Variance-Covariance } \\
\text { Matrix }\end{array}$ & $\left(\begin{array}{l}0.956 \\
-2.74 \times 10\end{array}\right.$ & $\left.2 \begin{array}{c}-2.74 \times 10^{-2} \\
2.66 \times 10^{-3}\end{array}\right)$ & $\left(\begin{array}{l}2.547 \\
-7.95 \times 10\end{array}\right.$ & $\left.\begin{array}{c}-7.95 \times 10^{-2} \\
4.61 \times 10^{-3}\end{array}\right)$ & $\left(\begin{array}{l}0.135 \\
-2.93 \times 10^{-}\end{array}\right.$ & $\left.\begin{array}{c}-2.93 \times 10^{-3} \\
2.41 \times 10^{-3}\end{array}\right)$ & $\left(\begin{array}{l}0.739 \\
-3.48 \times 10^{-}\end{array}\right.$ & $\left.\begin{array}{c}-3.48 \times 10^{-2} \\
4.02 \times 10^{-3}\end{array}\right)$ \\
\hline
\end{tabular}


Table 2. Estimates of Parameter of Exponential Distribution Using Data of $C P$ and $I P$ of $H Y_{a}$ and $H Y_{p}$ Groups of CABGPatients

\begin{tabular}{|c|c|c|c|c|c|c|c|c|}
\hline \multirow{3}{*}{ Parameter } & \multicolumn{4}{|c|}{$H Y_{a}$ Group } & \multicolumn{4}{|c|}{$H Y_{P}$ Group } \\
\hline & \multicolumn{2}{|c|}{$C P$} & \multicolumn{2}{|c|}{$I P$} & \multicolumn{2}{|c|}{$C P$} & \multicolumn{2}{|c|}{$I P$} \\
\hline & $\begin{array}{c}\text { Estimates/ } \\
\text { (t-ratio) }\end{array}$ & Gradients & $\begin{array}{c}\text { Estimates/ } \\
\text { (t-ratio) }\end{array}$ & Gradients & $\begin{array}{c}\text { Estimates/ } \\
\text { (t-ratio) }\end{array}$ & Gradients & $\begin{array}{c}\text { Estimates/ } \\
\text { (t-ratio) }\end{array}$ & Gradients \\
\hline$\alpha$ & $\begin{array}{c}4.71 \times 10^{-2} \\
(6.31)\end{array}$ & $2.09 \times 10^{-7}$ & $\begin{array}{c}4.74 \times 10^{-2} \\
(4.03)\end{array}$ & $1.85 \times 10^{-7}$ & $\begin{array}{c}8.15 \times 10^{-2} \\
(5.37)\end{array}$ & $-8.81 \times 10^{-8}$ & $\begin{array}{c}6.93 \times 10^{-2} \\
(3.61)\end{array}$ & $2.17 \times 10^{-7}$ \\
\hline Log-Likelihood & \multicolumn{2}{|c|}{1748.113} & \multicolumn{2}{|c|}{821.846} & \multicolumn{2}{|c|}{1465.947} & \multicolumn{2}{|c|}{$\begin{array}{c}1 \\
876.7729 \\
\end{array}$} \\
\hline Variance & \multicolumn{2}{|c|}{$9.26 \times 10^{-5}$} & \multicolumn{2}{|c|}{$2.22 \times 10^{-4}$} & \multicolumn{2}{|c|}{$1.46 \times 10^{-4}$} & \multicolumn{2}{|c|}{$3.02 \times 10^{-4}$} \\
\hline
\end{tabular}

Table 3. Survivalproportions $\hat{y}_{t}$ and $y / c$ of $C P$ and $I P$ respectively of Hypertension Absent $\left(H Y_{a}\right)$ and Present ( $H Y_{p}$ ) Groups of CABGPatients from Weibull and Exponential Distributions

\begin{tabular}{|c|c|c|c|c|c|c|c|c|}
\hline & \multicolumn{4}{|c|}{ Weibull Distribution } & \multicolumn{4}{|c|}{ Exponential Distribution } \\
\hline & \multicolumn{2}{|c|}{$H Y_{a}$} & \multicolumn{2}{|c|}{$H Y_{p}$} & \multicolumn{2}{|c|}{$H Y_{a}$} & \multicolumn{2}{|c|}{$H Y_{p}$} \\
\hline Years (t) & $\hat{y}_{t}$ & $9 / c^{c}$ & $\hat{y}_{t}$ & $9 / c$ & $\hat{y}_{t}$ & $9 / c_{t}$ & $\hat{y}_{t}$ & $y / c$ \\
\hline 0 & 1 & 1 & 1 & 1 & 1 & 1 & 1 & 1 \\
\hline 1 & 0.966 & 0.964 & 0.961 & 0.95 & 0.954 & 0.953 & 0.921 & 0.933 \\
\hline 2 & 0.928 & 0.922 & 0.906 & 0.89 & 0.910 & 0.909 & 0.849 & 0.870 \\
\hline 3 & 0.888 & 0.879 & 0.845 & 0.828 & 0.868 & 0.867 & 0.783 & 0.812 \\
\hline 4 & 0.848 & 0.835 & 0.783 & 0.767 & 0.828 & 0.827 & 0.721 & 0.757 \\
\hline 5 & 0.809 & 0.792 & 0.720 & 0.708 & 0.790 & 0.788 & 0.665 & 0.707 \\
\hline 6 & 0.770 & 0.751 & 0.659 & 0.651 & 0.753 & 0.752 & 0.613 & 0.659 \\
\hline 7 & 0.732 & 0.71 & 0.600 & 0.597 & 0.719 & 0.717 & 0.565 & 0.615 \\
\hline 8 & 0.696 & 0.671 & 0.543 & 0.546 & 0.686 & 0.684 & 0.520 & 0.574 \\
\hline 9 & 0.660 & 0.633 & 0.491 & 0.498 & 0.654 & 0.652 & 0.480 & 0.535 \\
\hline 10 & 0.626 & 0.597 & 0.441 & 0.454 & 0.624 & 0.622 & 0.442 & 0.499 \\
\hline 11 & 0.594 & 0.562 & 0.396 & 0.413 & 0.595 & 0.593 & 0.407 & 0.466 \\
\hline 12 & 0.562 & 0.529 & 0.354 & 0.375 & 0.568 & 0.566 & 0.375 & 0.435 \\
\hline
\end{tabular}

\subsubsection{Discussion}

The graphs in fig $1 \& 2$ of survival proportions obtained by using Weibull distribution of $C P$ and $I P$ for $H Y_{a}$ and $H Y_{p}$ groups of CABG patients shows that for $H Y_{a}$ group, the difference between the survival proportions of $C P$ and $I P$ is small at the start, continuously but slowly increasing, whereas the survival proportions of IP are lower than those of $C P$ and for $H Y_{p}$ group, the survival proportion of $I P$ are slightly lower at the start, almost equal from $6^{\text {th }}$ to $9^{\text {th }}$ year values and slightly higher at the end then those of $C P$.

The graphs in fig $3 \& 4$ of survival proportions obtained by using exponential distribution of $C P$ and $I P$ for $H Y_{a}$ and $H Y_{p}$ groups of CABG patients shows that when there is no hypertension, the difference is almost ignorable and when
$H Y_{p}$, the difference between the survival proportions of $C P$ and IP is continuously but slowly increasing, whereas the survival proportions of $C P$ are lo wer than those of $I P$.

The differences between the means $\mu_{C}$ and $\mu_{I}$ of survival proportions obtained by using Weibull and exponential distributions respectively of $C P$ and $I P$ respectively, for $H Y_{a}$ and $H Y_{p}$ groups of CABG patients are tested using $t$-statistic under the null hypothesis $\mathrm{H}_{\mathrm{o}}$ : $\mu_{\mathrm{I}}=\mu_{\mathrm{C}}$, against an alternative hypothes is $\mathrm{H}_{1}: \mu_{\mathrm{I}}>\mu_{\mathrm{C}}$. The values of $t$-statistic of $H Y_{a}$ and $H Y_{p}$ groups are $-0.344 \&-0.023$ and $0.031 \& 0.622$ (by weibull and exponential distributions respectively) when compared with $t_{0.05(11)}=1.796$, suggest that $H_{0}$ is accepted which 
means that the differences between the means of $C P$ and $I P$ of $H Y_{a}$ and $H Y_{p}$ groups of CABG patients, are statistically insignificant at $5 \%$ level of significance.

\subsubsection{Conclusion 1}

The differences between the means of survival proportions (obtained by using Weibull and exponential distributions) of $C P$ and $I P$ of $H Y_{a}$ and $H Y_{p}$ groups of CABG patients are statistically insignificant at $5 \%$ level of significance. This implies validity of using our new approach of formulation of complete population. Moreover, survival proportions of $H Y_{p}$ are lower than those of $H Y_{a}$, as observed by world over medical scientists.

\subsection{Diabetes Absent $\left(D_{a}\right)$ and Present $\left(D_{p}\right)$ Groups}

\section{(Male and Female CABG Patients)}

The survival proportions $\hat{y}_{t}$ and $\quad y / c$ of $C P$ and $I P$ respectively of $D_{a}$ and $D_{p}$ groups of CABG patients are obtained using Weibull Weibull and exponential distributions as explained earlier. The optimal estimates of parameters obtained by maximizing the log-likelihood function are given below in tables 4 and 5 .

The estimated values of scale parameter $\alpha>0$ and shape parameter $\beta>0$ for $C P$ and $I P$ of $D_{a}$ and $D_{p}$ groups of CABG patients are given in the tables $4 \& 5$ along with $t$-ratios in the parenthesis, indicating that the estimates of scale and shape parameters are significant at $5 \%$ level of significance. In case of Weibull Distribution the estimated value of $\beta$ is greater than 1 (for $C P$ and $I P$ of the $D_{a}$ and $D_{p}$ groups) which indicates increasing failure rate with time. The negative values of co-variances (for $C P$ and IP of $D_{a}$ and $D_{p}$ groups of CABG patients) indicates that the movements of $\hat{\alpha}$ and $\hat{\beta}$ are in the opposite directions.

Table 4. Est imat es of Parameters of Weibull Distribution Using Data of $C P$ and $I P$ of $D_{a}$ and $D_{p}$ Groups of CABGPatients

\begin{tabular}{|c|c|c|c|c|c|c|c|c|}
\hline \multirow{3}{*}{ Parameters } & \multicolumn{4}{|c|}{$D_{a}$ Group } & \multicolumn{4}{|c|}{$D_{p}$ Group } \\
\hline & \multicolumn{2}{|c|}{$C P$} & \multicolumn{2}{|c|}{ IP } & \multicolumn{2}{|c|}{$C P$} & \multicolumn{2}{|c|}{ IP } \\
\hline & $\begin{array}{c}\text { Estimates/ } \\
\text { (t-ratio) }\end{array}$ & Gradients & $\begin{array}{c}\text { Estimates/ } \\
\text { (t-ratio) }\end{array}$ & Gradients & $\begin{array}{c}\text { Estimates/ } \\
\text { (t-ratio) }\end{array}$ & Gradients & $\begin{array}{c}\text { Estimates/ } \\
\text { (t-ratio) }\end{array}$ & Gradients \\
\hline$\alpha$ & $\begin{array}{l}18.149 \\
(26.01) \\
\end{array}$ & $-2.48 \times 10^{-}$ & $\begin{array}{c}16.881 \\
(796.66) \\
\end{array}$ & $-2.17 \times 10^{-}$ & $\begin{array}{c}9.736 \\
(24.57) \\
\end{array}$ & $3.28 \times 10^{-10}$ & $\begin{array}{l}10.205 \\
(8.37) \\
\end{array}$ & $-1.37 \times 10^{-6}$ \\
\hline$\beta$ & $\begin{array}{c}1.154 \\
(27.83)\end{array}$ & $-1.36 \times 10^{-}$ & $\begin{array}{c}1.141 \\
(292.66)\end{array}$ & $-6.86 \mathrm{E}-06$ & $\begin{array}{c}1.380 \\
(20.07)\end{array}$ & $-3.91 \times 10^{-8}$ & $\begin{array}{c}1.249 \\
(22.96)\end{array}$ & $-8.1 \times 10^{-06}$ \\
\hline Log-Likelihood & \multicolumn{2}{|c|}{2688.629} & \multicolumn{2}{|c|}{1231.826} & \multicolumn{2}{|c|}{1023.245} & \multicolumn{2}{|c|}{450.3934} \\
\hline $\begin{array}{c}\text { Variance-Covar } \\
\text { iance } \\
\text { Matrix }\end{array}$ & $\begin{array}{l}0.487 \\
-1.45 \times 10^{-}\end{array}$ & $\left.\begin{array}{l}1.45 \times 10^{-2} \\
1.72 \times 10^{-3}\end{array}\right)$ & $\left(\begin{array}{l}1.487 \\
-4.81 \times 10^{-}\end{array}\right.$ & $\left.\begin{array}{l}-4.81 \times 10^{-2} \\
2.96 \times 10^{-3}\end{array}\right)$ & $\begin{array}{l}0.157 \\
1.01 \times 10^{-4}\end{array}$ & $\left.\begin{array}{l}1.01 \times 10^{-4} \\
4.72 \times 10^{-3}\end{array}\right)$ & $\begin{array}{l}0.74 \\
-4.26 \times 10\end{array}$ & $\left.\begin{array}{l}-4.26 \times 10^{-2} \\
7.90 \times 10^{-3}\end{array}\right)$ \\
\hline
\end{tabular}

Table 5. Estimates of Parameter of Exponential Distribution Using Data of $C P$ and $I P$ of $D_{a}$ and $D_{p}$ Groups of CABG Patients

\begin{tabular}{|c|c|c|c|c|c|c|c|c|}
\hline \multirow{3}{*}{ Parameter } & \multicolumn{4}{|c|}{$D_{a}$ Group } & \multicolumn{4}{|c|}{$D_{p}$ Group } \\
\hline & \multicolumn{2}{|c|}{$C P$} & \multicolumn{2}{|c|}{$I P$} & \multicolumn{2}{|c|}{$C P$} & \multicolumn{2}{|c|}{$I P$} \\
\hline & $\begin{array}{l}\text { Estimates/ } \\
\text { (t-ratio) }\end{array}$ & Gradients & $\begin{array}{c}\text { Estimates/ } \\
\text { (t-ratio) }\end{array}$ & Gradients & $\begin{array}{c}\text { Estimates/ } \\
\text { (t-ratio) }\end{array}$ & Gradients & $\begin{array}{c}\text { Estimates/ } \\
\text { (t-ratio) }\end{array}$ & Gradients \\
\hline$\alpha$ & $\begin{array}{c}5.07 \times 10^{-2} \\
(4.89)\end{array}$ & $1.36 \times 10^{-7}$ & $\begin{array}{c}5.05 \times 10^{-2} \\
(3.18)\end{array}$ & $3.53 \times 10^{-7}$ & $\begin{array}{c}0.1016 \\
(6.74)\end{array}$ & $-9.42 \times 10^{-7}$ & $\begin{array}{l}8.22 \times 10^{-2} \\
(3.99)\end{array}$ & $1.51 \times 10^{-7}$ \\
\hline Log-Likelihood & \multicolumn{2}{|c|}{2696.202} & \multicolumn{2}{|c|}{1235.46} & \multicolumn{2}{|c|}{1041.88} & \multicolumn{2}{|c|}{454.7762} \\
\hline Variance & \multicolumn{2}{|c|}{$6.45 \times 10^{-5}$} & \multicolumn{2}{|c|}{$1.57 \times 10^{-4}$} & \multicolumn{2}{|c|}{$3.58 \times 10^{-4}$} & \multicolumn{2}{|c|}{$6.76 \times 10^{-4}$} \\
\hline
\end{tabular}


The estimated survival proportions of $C P$ and $I P$ of $D_{a}$

Figure 6. Weibull Distribution and $D_{p}$ groups of CABG patients are given in table 6 and corresponding graphs (survival curves) in fig 5 to 8 .
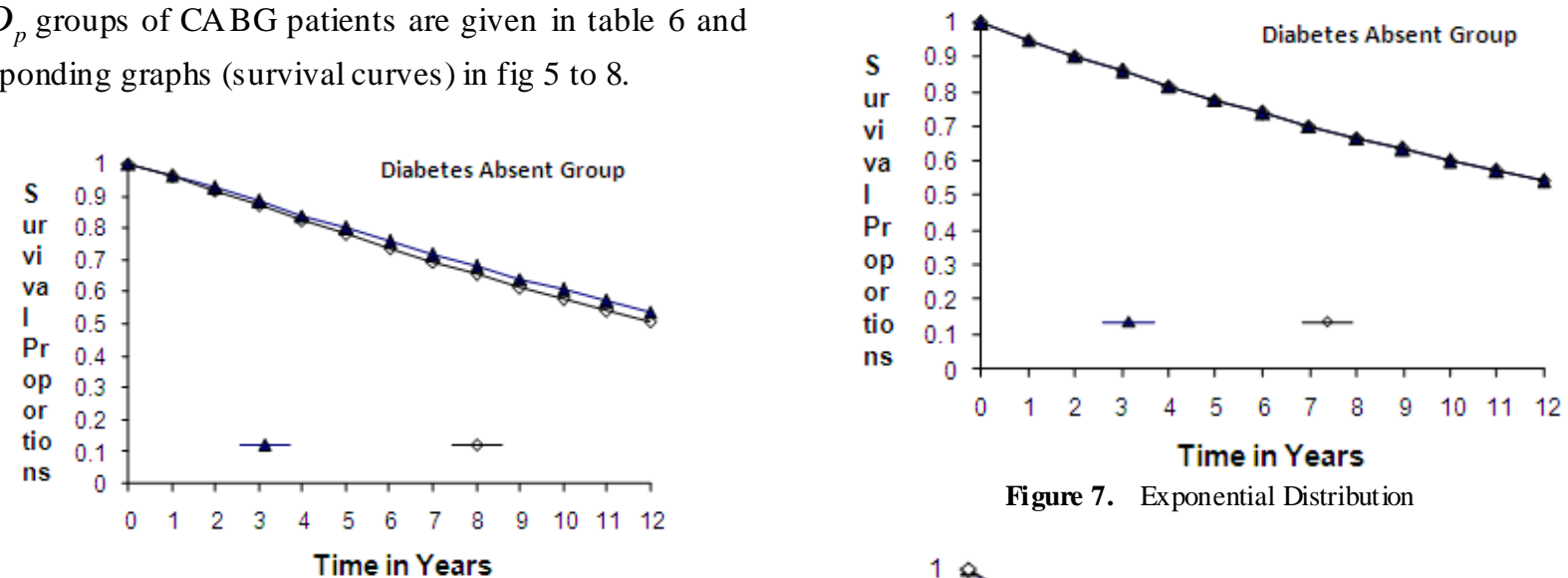

Figure 7. Exponential Distribution

Figure 5. Weibull Distribution

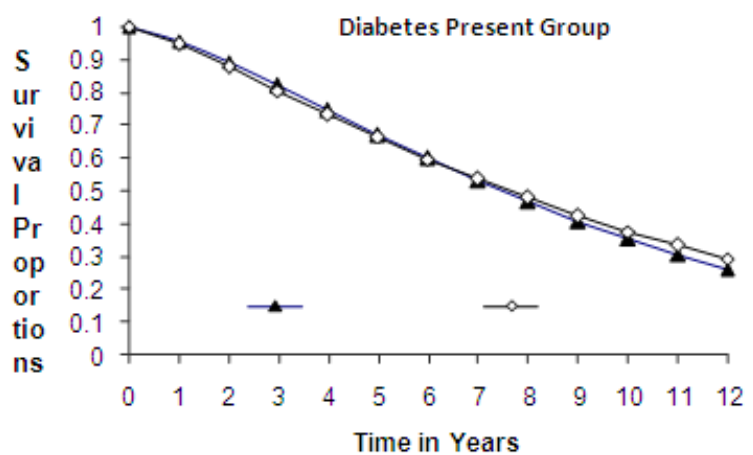

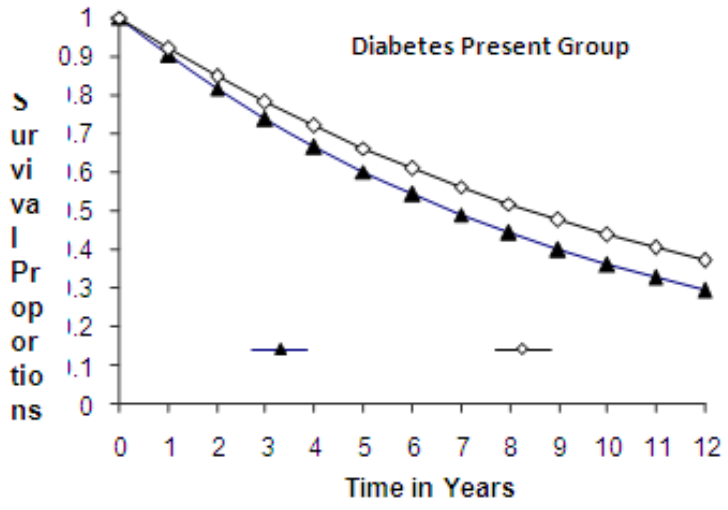

Figure 8. Exponential Distribution

Table 6. Survival Proportions $\hat{y}_{t}$ and $y / c$ of $C P$ and IP respectively of Diabetes Absent $\left(D_{a}\right)$ and Present $\left(D_{p}\right)$ Groups of CABG Patients from Weibull and

\begin{tabular}{|c|c|c|c|c|c|c|c|c|}
\hline & \multicolumn{5}{|c|}{ Weibull Distribution } & \multicolumn{3}{c|}{ Exponential Distribution } \\
\hline & \multicolumn{2}{|c|}{$D_{a}$} & \multicolumn{2}{c|}{$D_{p}$} & \multicolumn{2}{c|}{$D_{a}$} & \multicolumn{2}{c|}{$D_{p}$} \\
\hline \multirow{2}{*}{ Years (t) } & $\hat{y}_{t}$ & $9 / t^{2}$ & $\hat{y}_{t}$ & $9 / t^{\prime}$ & $\hat{y}_{t}$ & $9 / t^{\prime}$ & $\hat{y}_{t}$ & $9 / t^{c}$ \\
\hline 0 & 1 & 1 & 1 & 1 & 1 & 1 & 1 & 1 \\
\hline 1 & 0.965 & 0.961 & 0.957 & 0.947 & 0.950 & 0.950 & 0.903 & 0.921 \\
\hline 2 & 0.924 & 0.916 & 0.893 & 0.878 & 0.903 & 0.903 & 0.816 & 0.848 \\
\hline 3 & 0.882 & 0.87 & 0.821 & 0.805 & 0.859 & 0.859 & 0.737 & 0.781 \\
\hline 4 & 0.839 & 0.824 & 0.746 & 0.733 & 0.816 & 0.817 & 0.666 & 0.719 \\
\hline 5 & 0.797 & 0.779 & 0.671 & 0.664 & 0.776 & 0.776 & 0.601 & 0.662 \\
\hline 6 & 0.756 & 0.736 & 0.598 & 0.597 & 0.737 & 0.738 & 0.543 & 0.610 \\
\hline 7 & 0.716 & 0.694 & 0.530 & 0.536 & 0.701 & 0.702 & 0.491 & 0.562 \\
\hline 8 & 0.678 & 0.653 & 0.466 & 0.478 & 0.666 & 0.667 & 0.443 & 0.518 \\
\hline 9 & 0.640 & 0.614 & 0.407 & 0.425 & 0.633 & 0.634 & 0.400 & 0.477 \\
\hline 10 & 0.605 & 0.577 & 0.354 & 0.377 & 0.602 & 0.603 & 0.362 & 0.439 \\
\hline 11 & 0.570 & 0.542 & 0.306 & 0.334 & 0.572 & 0.573 & 0.327 & 0.404 \\
\hline 12 & 0.537 & 0.508 & 0.263 & 0.294 & 0.544 & 0.545 & 0.295 & 0.372 \\
\hline
\end{tabular}




\subsubsection{Exponential Distributions}

\section{Discussion}

The graphs in fig $5 \& 6$ of survival proportions obtained by using Weibull distribution of $C P$ and $I P$ for $D_{a}$ and $D_{p}$ groups of CABG patients shows that for $D_{a}$ group, the difference between the survival proportions of $C P$ and $I P$ is small at the start, continuously but slowly increasing, whereas most of the survival proportions of $I P$ are lower than those of $C P$ and for $D_{p}$ group, the survival proportions of $I P$ are slightly lo wer at the start, almost equal from $6^{\text {th }}$ to $7^{\text {th }}$ year values and slightly higher at the end than those of $C P$.

The graphs in fig 7\& 8 of survival proportions obtained by using exponential distribution of $C P$ and $I P$, for $D_{a}$ and $D_{p}$ groups of CABG patients shows that when there is no diabetes, the difference is almost ignorable and when $D_{p}$, the difference between the survival proportions of $C P$ and $I P$ is continuously but slowly increasing, whereas the survival proportions of $C P$ are lower than those of $I P$.

The differences between the means $\mu_{C}$ and $\mu_{I}$ of survival proportions (obtained by using Weibull and distributions respectively) of $C P$ and $I P$ respectively of $D_{a}$ and $D_{p}$ groups of CABG patients, are tested using $t$-statistic under the null hypothes is $\mathrm{H}_{\mathrm{o}}: \mu_{\mathrm{I}}=\mu_{\mathrm{C}}$, against an alternative hypothesis $\mathrm{H}_{1}: \mu_{\mathrm{I}}>\mu_{\mathrm{C}}$. The values of $t$-statistic of $D_{a}$ and $D_{p}$ groups are-0.329\&-0.050 and $0.012 \& 0.786$ (by weibull and exponential distributions respectively) when compared with $t_{0.05(11)}=1.796$, suggest that $\mathrm{H}_{0}$ is accepted which means that the differences between the means of $C P$ and IP of CABG patients groups of $D_{a}$ and $D_{p}$, are statistically insignificant at $5 \%$ level of significance.

\subsubsection{Conclusion 2}

The differences between the means of survival proportions (obtained by using Weibull and exponential distributions) of $C P$ and $I P$ of $D_{a}$ and $D_{p}$ groups of CABG patients are statistically insignificant at $5 \%$ level of significance. This implies validity of our new approach of comp lete population. Moreover, survival proportions of $D_{p}$ are lower than those of $D_{a}$, as observed by all medical scientists.

\section{Final Conclusions}

Summing up the conclusions 1 and 2, finally, as a whole the differences between the means of survival proportions (obtained by using Weibull distribution and exponential distributions) of $C P$ and IP of CABG patients groups (hypert ension and diabetes) are statistically insignificant at $5 \%$ level of significance. This implies complete population from its incomp lete population as proposed in our article[21] may be used for better medical investigations. The survival proportions of $H Y_{p}$ and $D_{p}$ are lower than those of $H Y_{a}$ and $D_{a}$ respectively, whereas survival proportions of $D_{p}$ are comparatively lowest, as observed by world over medical scientists. Thus, the CABG patient's data has been adequately modeled by both distributions (Weibull and exponential).

Moreover, forecasting of the survival proportions of the CABG patients is also possible by Bayesian analysis (Kalman Filter approach) as advocated by Meinhold and Singpurwalla in 1983 in an American journal.

\section{REFERENCES}

[1] Abernathy, R. B. (1998), The New Weibull Handbook. 3rd ed. SAE Publications, Warrendale.PA.

[2] American Heart Association Dallas, Texas (2007). Heart Disease and Stroke Statistics.

[3] Axel Vise, Yiwen Zhu, Dalit May, Veena Afza, Elaine Gong, Catia Attanasio, Matthew J. Blow, Jonathan. C. Cohen, Edward M. Rubin \& Len A. Pennacchio (2010). Targeted deletion of the 9p21 non-coding coronary artery disease risk interval in mice.

[4] Bain, L.J. and Englehardt, M. (1991), Statistical Analysis of Reliability and Life-Testing Models: Theory and Methods, 2nd ed., Marcel Dekker, New York.

[5] Bunday, B.D. and Al-Mutwali, I.A (1981). Direct optimization for calculation maximum likelihood estimates of parameters of the Weibull distribution, IEEE Trans, Reliability, R-30, No.4, 367-339.

[6] Cohen. A,C (1965).Maximum likelihood estimation in the Weibull distribution based on complete and on censored samples. Technometrics.Volume.7.no.4.579-588

[7] Crow, L.H. (1982), "Confidence Interval Procedures for the Weibull Process With Applications to Reliability Growth," Technometrics, 24(1):67-72.

[8] Cyrus R Mehta (1981). Sequential comparison of two exponential distributions with censored survival data. Biometrika 68(3): 669-675.

[9] David G. Kleinbaum, Mitchel Klein (2005). Survival analy sis: a self-learning text.

[10] Dorlands Medical Dictionary (2009): Coronary Artery Disease.

[11] Goldstein. L, Adams R, Alberts. M. L. Appel, L. Brass, C. Bushnell, A. Culebras, T. DeGraba, P. Gorelick, J. Guyton (2006). American Heart Association; American Stroke Association Stroke Council. Primary Prevention of Ischemic Stroke, American Journal of Ophthalmology: American Heart Association. , Volume 142, Issue 4, 716-716. 
[12] Gross A J and Clark V A (1975). Survival Distribution: Reliability Applications in the Biomedical Sciences Wiley.

[13] Hansson Göran K, M.D. (2005). Inflammation, Atherosclerosis, and Coronary Artery Disease. Volume 352:1685-1695, Number 16.

[14] Heart and Stroke Foundation Canada (1997). Heart Disease and Stroke Statistics.

[15] Heymann C.Von Heymann (2002). Successful treatment of refractory bleeding with recombinant factor VIIa after redoes coronary artery bypass graft surgery. Journal of Cardiothoracic and VascularAnesthesia, Volume 16, Issue 5, 615-616.

[16] Hisada \& Arizino (2002).Reliability tests for Weibull distribution with vary ing shap e-p arameter, based on complete data Reliability, IEEE Transactions Volume 51, Issue 3, Sep: $331-336$.

[17] Jennifer Heisler, RN (2008) After Coronary Artery Bypass Graft Surgery-Recovering From Open Heart Surgery.

[18] John H. Lemmer (2003). Hand Book of Patient Care in Cardiology Surgery; Lippincott Williams \& Wilkins.

[19] Kaplan.E.L.Meier.P. (1958). Nonparametric estimations from incomplete observations.

[20] Khan. K.H. and Mahmud Z (1999). Weibull Distribution Model for The Breast Cancer Survival Data Using Maximum Likelihood Method.J.American.Assoc.53, 457 - 481.

[21] Khan K..H, Saleem M and Mahmud. Z. (2011). Survival Proportions of CABG Patients: A New Approch. Volume 3, Number 3.

[22] Klein.P.J and Moeschberger.L.M (1997, 2003). Survival Analysis Techniques for Censored and Truncated Data.

[23] Lang Wu (2010). Mixed effects models for complex data.

[24] .Lanju Zhang and William F. Rosenberger (2007). Response-adaptive randomization for survival trials: the parametric approach. Journal of the Roy al Statistical Society: Series C (Applied Statistics). Volume 56, Issue 2, pages 153-165.
[25] Lawless Jerald F. Lawless (1982, 2003). Statistical Models and Methods for Lifetime Data, John Wiley and Sons, Inc New York.

[26] Lawrence M.Leemis (1995). Reliability Probabilistic Modela and Statistical Methods.

[27] Lee Jaey ong, Kim Jinseog and Jung Sin-Ho (2006). Bay esian analysis of paired survival data using a bivariate exponential distribution. Lifetime Data Analysis.Volume 13, Number 1.

[28] Maan, N. R, Schafer, R.E and Singpurawalla, N.D. (1974). Method for Statistical Analysis of reliability and Lifetime Data. New Yourk: Wiley.

[29] Nelson..W, (1982). Applied Life Data Analysis. Newy ork:Wiley .

[30] Rao Venkata and Kiran Ravi (2011). Evaluation of correlation between oxidative stress and abnormal lipid profile in coronary artery disease.J Cardiovasc Dis Res. Jan-Mar; 2(1): 57-60.doi: 10.4103/0975-3583.78598.

[31] Saleem M, Mahmud. Z and Khan.K.H (2012) Maximum Likelihood Estimation of CABG Patients By Parametric Models Based on Incomplete and Complete Population. American Journal of Statistics and Mathematics (JOSAM): Bioinfo Publications.

[32] Sridhar, K.N.; Mun Choon Chan (2009).Modeling link lifetime data with parametric regression models in MANETs. IEEE. Volume: 13 Issue:12

[33] Sun Zhonghua and Hong. Ng. Kwan- (2011). Coronary computed tomography angiography in coronary artery disease. World J Cardiol. 2011 September 26; 3(9): 303-310.

[34] Swaminathan R and Brenner H. (1998, 2011). Statistical methods for Cancer Survival Analysis Vol 1 and Vol 2.

[35] William S. Weintraub MD; Ellis, L. Jonees, MD; Josef M. Craver. MD; Ralph Grossedwald, BS; Robert A.Guyton,MD, $(1995,1997)$. In Hospital and Long term Outcome After Re-cooperative Coronary Artery Bypass Graft Surgery. American Heart Association.

[36] William S. Weintraub, MD; Stephen D. Clements Jr, MD; L. Van-Thomas Crisco, MD; Robert A. Guyton,ND (2003). Twenty years Survival After Coronary Artery Surgery. American Heart Association. 